\section{IN THE NEWS}

Promise of therapy Using human embryonic stem (ES) cells in research is controversial, and adult stem cells have been proposed as a potential alternative. This debate has been fuelled by recent landmark experiments that bring us closer to stemcell therapy. Catherine Verfaillie's group found adult stem cells in mice and men "that [seem] to have all the versatility of embryonic stem cells" (The New York Times), overturning "the dogma that animal development proceeds in one irreversible direction, from the unspecialized [zygote] to the highly specialized cells of an adult body" (Washington Post). When injected into early mouse embryos, these adult stem cells that come from bone marrow contribute to many tissue types and can make up to $40 \%$ of all cells. Importantly, they "do not form a spontaneous tumor known as a teratoma [...] and they could in many cases be derived from the patient" (The New York Times), eliminating a risk of immune rejection.

So has ES-cell research become superfluous? Those who "have been battling to end all human embryonic research because it requires the destruction of five-dayold embryos" (Financial Times) might argue that it has; however, experiments from Ron McKay's group, published at the same time prove otherwise. "McKay's team was able to turn mouse [ES] cells into dopamineproducing neurons - the kind that would be needed to correct the devastating effects of Parkinson's disease" (New Scientist). It is not clear how close we really are to stem-cell-based therapy. Although some say that "clinical trials could begin in 12 to 18 months" (The New York Times), others wonder "how scientists would be able to produce enough adult cells in the laboratory to use widely for medical treatments" (Financial Times).

RNA PROCESSING

\title{
Skipping between alternatives
}

Up to $30 \%$ of mutations that cause human diseases are believed to generate in-frame nonsense codons. How the cell handles such deleterious transcripts can determine the severity of disease. Cells are known to neutralize these transcripts in two ways: by triggering nonsense-mediated decay (NMD), a translationdependent process that degrades transcripts containing premature truncation codons (PTCs), or nonsense-associated alternative splicing (NAS), in which offending exons are removed before splicing by a putative nuclear reading-frame scanning mechanism (see figure).

This scanning mechanism, however, runs counter to a tenet of cell biology - that splicing and translation occur in different cellular compartments. As a result, it's been argued that NAS occurs when any mutation disrupts exonic signal enhancer (ESE) sequences, which are cryptic exonic signals that determine the splicing of certain exons. Caputi et al. now provide evidence for this theory with the finding that exon skipping in the fibrillin 1 (FBN1) gene, which causes Marfan syndrome (MFS), is caused by the disruption of an ESE. Conversely, Wang et al. report that exon skipping in the T-cell receptor- $\beta$ (TCRB) gene occurs specifically in response to nonsense mutations, regardless of their exonic location, so arguing against the ESE-disruption model. This work hints at a controversial mode of gene regulation that requires nuclear translation.

Caputi et al. studied an FBN1 mutation (Y2113X), which induces the skipping of exon 51 in MFS patients, and found that the mutant transcript underwent both NMD and exon skipping in a patient's fibroblasts. However, although NMD was abrogated by translation inhibition in these cells, exon skipping remained unaffected, indicating that these processes are independently activated. The authors next altered the position of exon-51 PTCs in an FBN1 minigene, reasoning that if a nuclear-scanning mechanism was causing exon skipping, it should be unaffected by a PTC's position. However, they found that exon skipping only occurred when mutations were in a particular region of exon 51 - a region that behaved as an ESE in splicing assays.

Given these results, those of Wang and colleagues are somewhat surprising. They focused on the TCRB gene because it commonly acquires PTCs during VDJ-region rearrangements in T-cell development, generating mutant transcripts that are degraded by NMD. Here they report that an alternative (alt-)mRNA, in which the mutation-bearing exon is skipped, is upregulated severalfold in response to PTCs in the VDJ exon. To test whether this is due to the disruption of an ESE, the authors assayed the effects on splicing of placing different point mutations at various positions in this exon. They found that nonsense codons at all four positions tested caused exon skipping, whereas missense and silent mutations in these positions did not.

So what might mediate this response? Wang et al. controversially propose that it requires a translationlike mechanism because, in their study, exon skipping required a start codon to define the reading frame. Moreover, a stem-loop sequence that blocks translation introduced into the 5 ' leader exon prevented alt-mRNA upregulation. And finally, this response was inhibited by tRNA suppressors.

Wang et al. propose different models for how this translation-like scanning mechanism might operate, both in the nucleus and in the cytoplasm. Although preliminary evidence of nuclear translation has been reported, this paper will no doubt create much debate in the field, and will hopefully create more data to clarify how nonsense codons are processed to shed new light on how these responses can alter the severity of genetic disease.

(4) References and links

Jane Alfred

ORIGINAL RESEARCH PAPERS Caputi, M. et al. A nonsense mutation in the fibrillin-1 gene of a Marfan syndrome patient induces NMD and disrupts an exonic splicing enhancer. Genes Dev. 16, 1754-1759 (2002) | Wang, J. et al. Alternatively spliced TCR mRNA induced by disruption of reading frame. Science 297, 108-110 (2002)

FURTHER READING Cartegni, L. et al. Listening to silence and

understanding nonsense: exonic mutations that affect splicing. Nature Rev. Genet. 3, 285-298 (2002)

WEB SITES

Karen Beemon's laboratory:

http://www.bio.jhu.edu/Directory/Faculty/Beemon/Default.html Miles Wilkinson's laboratory:

Miles Wilkinson's laboratory:
http://www3.mdanderson.org/ immuno/DEPARTMENT/wilkinson.html

Modified from L. Cartegni et al. ๑ (2002) Macmillan Magazines Ltd.

\section{Nuclear scanning}

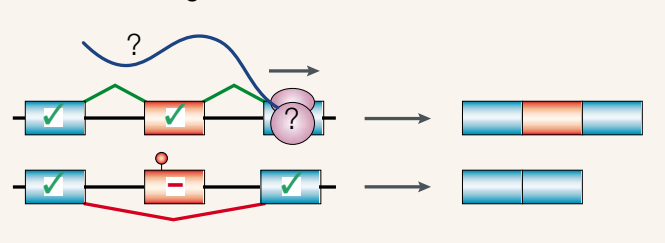

ESE disruption

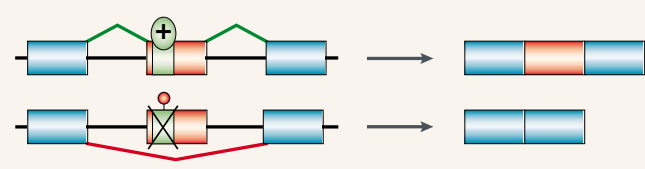

$\begin{array}{lll}\square \text { Exon } & \sim \text { Polypeptide } & \square \text { Intact reading frame } \\ \square \text { ESE } & \text { ? } \begin{array}{l}\text { Nuclear translation- } \\ \text { like machinery }\end{array} & \begin{array}{l}\text { Interrupted reading } \\ \text { frame }\end{array} \\ & & \text { P PTC }\end{array}$

